\title{
Anesthesia for Trauma: New Evidence and New Challenges
}

\author{
Corey S. Scher (Ed). Springer, 2014. ISBN 978-1-4939-0908-7
}

\author{
Doreen Yee, MD
}

Received: 17 February 2015/Accepted: 9 March 2015/Published online: 17 March 2015

(C) Canadian Anesthesiologists' Society 2015

Care of the severely injured trauma patient requires integrated care provided by a high-functioning trauma team. Anesthesiologists, with their attendant skill sets in airway management, resuscitation, and vascular access, are uniquely positioned to be essential members, if not leaders, of this team. These skills sets frequently need to be "modified or adapted" to the trauma population, which is a very heterogeneous group. Corey S. Scher, the editor of this book, attempts to provide the necessary information to achieve this end.

One strong point of this multi-author textbook, which is not captured in the "trauma chapter" of most major anesthesia textbooks, is the valuable detailed coverage of most of the spectrum of trauma care that our specialty will encounter. A major weakness, however, is the lack of meticulous editing. For example, there are numerous typographical errors in more than half of the chapters, ranging from spelling errors and incorrect wording to incomplete sentences. This can become increasingly annoying while reading through the subject matter.

Some chapters, e.g., the first chapter, deliver a good summary or overview of trauma care, while others state specific considerations for special situations. In addition, other chapters discuss controversial areas and newer ideas which have resulted in changes in practice by some practitioners. The second chapter, which deals with airway management, is well written and comprehensive and includes references to special trauma airway situations as well as the role of newer airway equipment.

D. Yee, MD ( $\varangle)$

Department of Anesthesia, University of Toronto, Toronto, ON, Canada

e-mail: Doreen.Yee@sunnybrook.ca
The third chapter, "Physiological Derangement of the Trauma Patient", is a noteworthy topic, though the chapter suffers badly from the previously mentioned editing deficiencies. For instance, the word "hyperfibrihock" was used instead of "hyperfibrinolysis". Furthermore, the two tables on hemorrhagic shock (Tables 3.2 and 3.3) have been abbreviated almost to the point of being unintelligible. There are also notable omissions. For example, the use of etomidate is not mentioned in the discussion on acute adrenal insufficiency, and there is no mention of surgical rib fixation in patients with significant flail chest, which could lead to fewer ventilator and intensive care unit days. Another weakness of the chapter is the failure to link the results of referenced animal studies to knowledge that can be applied to relevant clinical situations in trauma. As a multi-authored chapter, with members in the group possibly less familiar with trauma patients, it fails to achieve what Dr. Scher conveys in his preface, "a consensus of practice is offered in each chapter".

The fourth chapter, "Blood Transfusion and Coagulation Disorders" is a good summary of the topic, although it is arguably inappropriate to include the final point, "achieve and maintain deep anesthesia", in Table 4.1 as one of the Principles of Early Resuscitation. It may be an intriguing idea, but there is minimal evidence for this practice, and it may confuse trainees. The next chapter on "General Principles of Intraoperative Management..." has some good practical points, but once again, the lines are blurred between currently accepted standards of care and what the author and his centre consider as new concepts that will become the standard of care. Many clinicians would use vasopressin to maintain blood pressure as part of resuscitation during a massive transfusion scenario. Nevertheless, a few 
eyebrows would now be raised at the advice to use vasopressin to start the resuscitation of a severe trauma patient in the operating room in order to "diminish the size of the circulation" and decrease the amount of blood products /fluids given. The concept of "pressure-support resuscitation" is intriguing but, to date, based mostly on animal studies. Furthermore, the goal of this action somewhat contradicts the goal of "maintaining deep anesthesia" to improve tissue perfusion as suggested in the previous chapter.

The two chapters dealing with acute and chronic pain are excellent summaries of pain issues experienced by various trauma patients, as are the majority of the seven chapters on injuries to body systems. Several controversial areas are addressed and discussed. The chapter on brain injury provides an overview of the current literature and ties it to treatment recommendations. In the section on spinal cord injury, there is an excellent "common sense" discussion of airway management and the use of cricoid pressure in patients with suspected spine injury. A comprehensive chapter on thoracic and abdominal injuries is presented complete with sections on damage control surgery and damage control resuscitation strategies. The two chapters on burn injury physiology and anesthetic management offer a welcome summary that is rarely found in major anesthesia texts, although the chapters on facial and ocular trauma and musculoskeletal injury and microvascular surgery were disappointing. In particular, the chapter on facial and ocular trauma was superficial and focused more on pediatric facial injuries, which actually comprise a minority of all facial injuries. There is no discussion of awake intubation techniques for acute airway management and no mention that most isolated mandibular fractures (after mechanical temporomandibular joint obstruction is ruled out) become uncomplicated airways after the trismus is resolved with muscle relaxants. The chapter on musculoskeletal injuries is mainly about microvascular surgery and anesthetic considerations. Realistically, only a small percentage of trauma patients require muscle flaps, even though the majority have some kind of extremity fracture. It would be useful to address a few more areas in orthopedic trauma, such as damage control orthopedic surgery, management of bleeding patients with complex pelvic fractures, and reasons for early long bone fixation.

The next section of the text focuses on special patient populations. The chapter on pediatric trauma provides a basic review of the principles of the Advanced Trauma Life Support program and anatomic and physiologic differences between children and adults, and it includes an extensive discussion about standard monitors. A focus on the differences in injury patterns of children (e.g., from car seats, battered children syndrome, etc.) as related to their anatomy would have been useful. In contrast, the chapter on trauma in pregnancy was outstanding; it addressed specific injuries in pregnancy and the effects of maternal physiologic changes on treatment. In some respects, the geriatric trauma chapter was somewhat outdated, for instance, ketamine is no longer contraindicated in head injury, and fresh frozen plasma would not be used aggressively to correct bleeding from warfarin when prothrombin complex concentrates have been available for some time.

The final few chapters provide different perspectives on trauma care from the critical care arena and give the anesthesiologist an idea of the importance of the resuscitation continuum. Chapters 20 and 21 emphasize the military influence on civilian trauma systems and thus offer interesting insight.

In summary, this is a good first attempt to cover an important area of clinical anesthesia in greater detail than is offered by major textbooks. The challenges of a multiauthored textbook are present in the obvious repetition and inconsistency in some areas. Improved editing in future editions should make for smoother reading.

Conflicts of interest None declared. 